\title{
Conceptual Determination and Assessment of Cyanosis
}

\author{
N. F. Azmi1,2*, F. Delbressine ${ }^{1}$, L. Feijs ${ }^{1}$ \\ ${ }^{1}$ Eindhoven University of Technology, Designed Intelligence group, Eindhoven, The Netherlands. \\ 2 Faculty of Electronics and Computer Engineering, Computer Engineering Department, Universiti Teknikal \\ Malaysia Melaka, Hang Tuah Jaya, Durian Tunggal, Malacca 76100, Malaysia. \\ * Corresponding author. Tel.: +31645583125; email: n.f.b.azmi@tue.nl \\ Manuscript submitted April 29, 2016; accepted July 10, 2016. \\ doi: 10.17706/ijbbb.2016.6.3.75-83
}

\begin{abstract}
Cyanosis can be detected in a blue-purplish discoloration area commonly around the mucous membranes which are around the lips, fingers and toes. It is one of the respiratory distress' symptoms in newborn babies that can be caused by either the low level of oxygenated blood or poor circulation. Low oxygen saturation in the blood will make the skin and lips appear to be blue since the blood's color changes from bright red to a darker bluish. Babies with cyanosis need urgent assessment and initiation of medical care. Therefore, a systematic and rational approach in identifying cyanosis symptoms are crucial since its occurrence is known to be one of the most difficult situation to be handled in pediatric world. Thus, the early detection of cyanosis symptoms and understanding the physiology of oxygenation in neonates are important to save the babies' lives. The goals of this paper are to introduce a new approach for early cyanosis detection and determination of cyanotic and non-cyanotic skin color based on the actual reflectance spectrum of oxyhemoglobin, $\left(\mathrm{HbO}_{2}\right)$ and deoxyhemoglobin, $(\mathrm{Hb})$. The actual transmission spectrum and the wavelength of cyanotic and non-cyanotic skin are calculated based on optical properties in each skins' layers. A block diagram is developed to represent the light's pathway in the cyanotic and non-cyanotic skin color and the transfer functions in each block diagram used to describe the relationship between individual layers. Finally, as an objective measure of cyanotic and non-cyanotic skin color, the International Commission on Illumination (CIE) of color space values $\left(\mathrm{L}^{*}, \mathrm{a}^{*}\right.$ and $\left.\mathrm{b}^{*}\right)$ are determined. The calculation results reveal that colorimetry assessment can demonstrate the evaluation of cyanosis based on oxygenation level in the blood.
\end{abstract}

Key words: Cyanosis, neonate, modeling, biological database.

\section{Introduction}

Cyanosis is derived from the Greek word "kuaneos" which means dark blue. It refers to the blue purplish discoloration commonly seen in mucous membranes [1], [2] for example around the lips, fingers and toes. Cyanosis is one of the common symptoms of respiratory distress in a newborn baby. Researchers highlighted that if a baby's skin or lips turn blue, it is always referred to a low level of oxygenated blood or poor circulation [2], [3]. When oxygen level in blood decreases, the blood color changes from bright red to a darker bluish and these can make the skin and lips appear to be blue.

Central cyanosis is often visually recognized by dark-blue or purple discoloration of the tongue and the linings of the mouth shown in Fig. 1 [4]. The skin color changes is one of the observationational elements the midwives will look for from 1 to 5 minutes after birth [5]. Central cyanosis is usually caused by diseases 
of the heart or lungs. The lack of hemoglobin in the blood can be measured using a pulse oximetry, a standard tool used for measuring the saturation of oxygen in hemoglobin. The oxygen saturation can be estimated with reasonable accuracy for an oxygen saturation of $60 \%$ or greater [1].

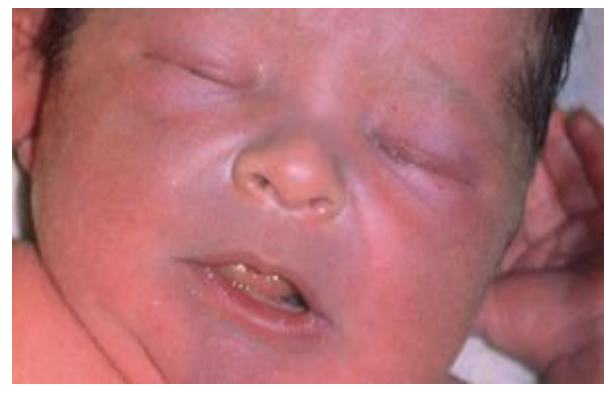

Fig. 1. Central cyanosis [5].

There are two states of hemoglobin: $\mathrm{HbO}_{2}$ and $\mathrm{Hb}$. The concentration of reduced hemoglobin is an important factor of the cyanosis to occur. It also is known as deoxygenated hemoglobin [6]. The cyanosis may become apparent when the amount of deoxygenated hemoglobin is as little as 3 to $5 \mathrm{~g} / \mathrm{dL}$. According to [1], [7], for a normal healthy newborn, the hemoglobin count is $18 \mathrm{~g} / \mathrm{dL}$. Here, an amount of $15 \mathrm{~g} / \mathrm{dL}$ $(18-3 \mathrm{~g} / \mathrm{dL})$ is the amount of hemoglobin that is saturated with oxygen. For example, $15 \mathrm{~g} / \mathrm{dL}$ indicated the amount of hemoglobin saturated with oxygen. Thus, cyanosis can be detected when $83 \%$ or less of oxygen saturation $\left(\mathrm{SaO}_{2}\right)$ is detected for instance by using a pulse oximetry. Accessing cyanosis in a newborn is highly subjective, and the evaluation of cyanosis is complicated because of many factors such as a surrounding's illuminant color and its intensity and the influences of the color of objects in the environment [8]. Therefore, the goal of this paper is to introduce a new approach for early cyanosis detection and determination of cyanotic and non-cyanotic skin color. The methods are based on the actual reflectance spectrum of $\mathrm{HbO}_{2}$ and $\mathrm{Hb}$, and CIE L*a*b* values when the skin appeared as cyanotic or non-cyanotic skin are determined.

\section{Theoretical Foundation}

\subsection{Observer Model of Cyanotic Skin}

An observer model which gives a cyanosis overview can be modeled using the optical properties of human skin layers. As shown in Fig. 2., the optics of human skin can be divided into two parts which are the boundaries between two materials and the material properties of each layer. Boundary 1 refers to the boundary between the air and the skin layer (epidermis) and the Boundary 2 refers to the boundary between the epidermis and the dermis. Several components may describe the light propagation in the skin layers including transmission, absorption and scattering in Fig. 2. and it depends on the properties and the pigments in the boundary and each layer. An incoming incident radiation, $\mathrm{I}_{\mathrm{In}}$, is shown together with a reflected rays of Ray 1 and Ray 2, and a transmitted ray of Ray 3. $I_{I n}$ is the refractive index of air; $n_{2}$ is the refractive index of skin while $\mathrm{n}_{3}$ is the refractive index of red blood cell (RBC).

\subsection{Series of Transfer Function in Skin's}

The observer model in Fig. 2 can be represented in the next series of the transfer function in the block diagrams as illustrates in Fig. 3. The block diagrams and the transfer functions in this chart described the relation between the input and outputs of the light optical pathways in human skin as well as to observe the cause and effect throughout the system. The system transfer function of the epidermis, $\mathrm{HbO}_{2}, \mathrm{Hb}$, dermis and hypodermis are labeled as $H_{E p i}, H_{H b 02}, H_{H b}, H_{\text {Dermis }}$ and $H_{H y p o d e r m i s}$, respectively. $I_{I n}$ is referred to incoming 
light while Ray 1, Ray 2 and Ray 3 are the output rays of the light coming out from the skin. As observed from the block diagrams, there are three different output rays can explain the light paths in the human's skin.

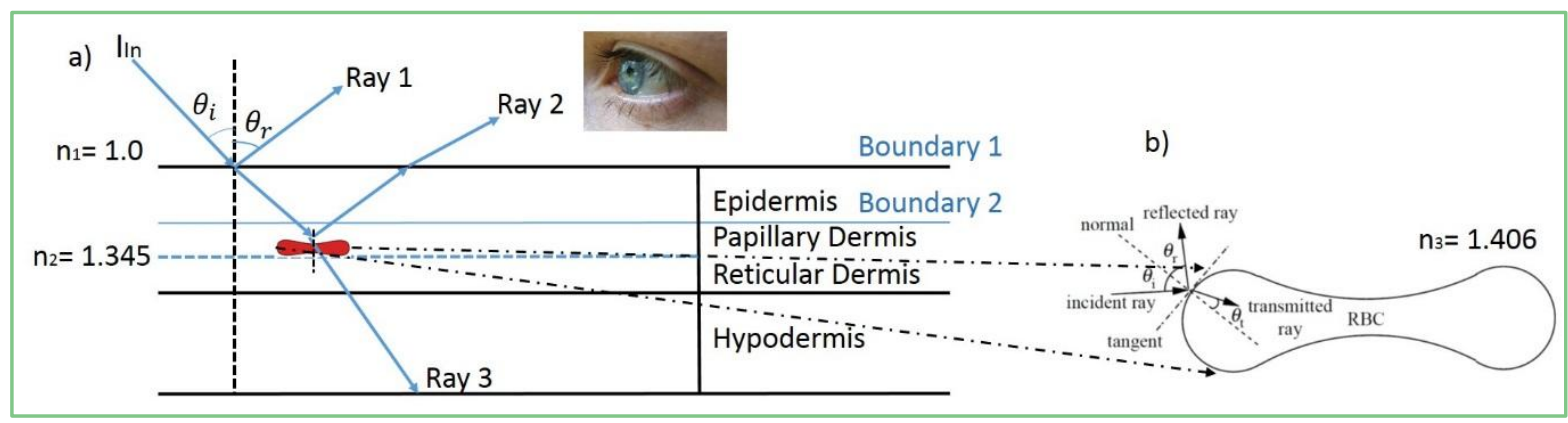

Fig. 2. a) A sketch of human skin and the optical properties of the skin layers. b) The terminology for Fresnel's equation for an incident ray that propagates in a scattering cell [9].

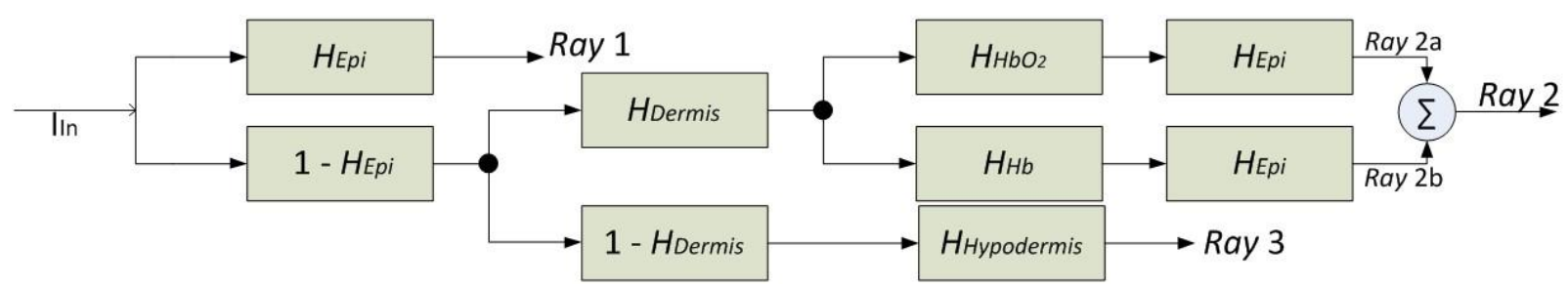

Fig. 3. Block diagrams of transfer function in skin optic.

The first transfer function, $H_{E p i}$ is referred to the transfer function of light in the epidermis. The incident light is partially reflected as a Ray 1 and partially transmitted to the dermis. The output of Ray 1 can be defined in term of $H_{E p i}$ as

$$
\operatorname{Ray} 1=H_{E p i} * I_{I n}
$$

The fraction of light which continues to travel to the dermis and meet the RBCs will be back- scattered and return into the upper layer; back to the transfer function of the epidermis, $H_{E p i \text {. The reflected ray of Ray }}$ 2 in Fig. 3 can be obtained by Ray $2=$ Ray 2a + Ray 2b where Ray 2a and Ray 2b are derived in the following equations respectively.

$$
\begin{gathered}
\text { ay } 2 a=H_{E p i} * H_{H b O 2} *\left(1-H_{E p i}\right) * I_{I n} \\
\text { Ray } 2 b=H_{E p i} * H_{H b} *\left(1-H_{E p i}\right) * I_{I n}
\end{gathered}
$$

In the last layers of skin, the light continue to be transmitted to the final of the transfer function of hypodermis, $H_{\text {Hypodermis. }}$ The Ray 3 and in Fig. 3 . can be obtained by the following transfer functions' equation.

$$
\text { Ray } 3=H_{E p i} *\left(1-H_{\text {Dermis }}\right) *\left(1-H_{E p i}\right) * I_{I n}
$$

\section{Result and Discussion}


The bluish cyanosis was seen because the brain processed the light interaction upon the skin as those perceived by the optical pathways on human skin as derived in Fig. 2. Eyes need three parameters which are the observer, the illuminant of a light source and the reflectance spectral of the object viewed to see colors. Based on the transfer function given in Eq.2 and Eq.3, the blue color of cyanosis can be can be quantified by calculating the incoming light source interacting with the transfer of $H_{H_{b 0}}$ and $H_{H b}$ and the color matching functions from the observer before the colors interpreted by the brain.

\subsection{Human Eye Sensitivity and Color Matching}

The two types of photoreceptors are rods and cones and each photoreceptor in human retina absorbs light and produces a neural (electrical) signal. The signals from all of the photoreceptors are processed within the eye and are processed within the eye while the brain produces the visual sensation. Because of the sensitivity to low levels of light, the rods are not omitted in color vision, whereas the cones are sensitive to higher levels of light, and the color vision is caused by the cone sensitivity in the eyes with approximately 100 million rods and 5 million cones are contained in the eyes. There are three types of primary cones; long-wavelength (L), medium-wavelength (M), and short-wavelength (S) cones. Each of them contains a pigment that absorbs light within a specific range of wavelengths [10]. At that time, the spectral sensitivities of the cones were yet to be known, but a color could be matched by the combination of three primaries, which could be used to specify color (Maxwell, 1860s).

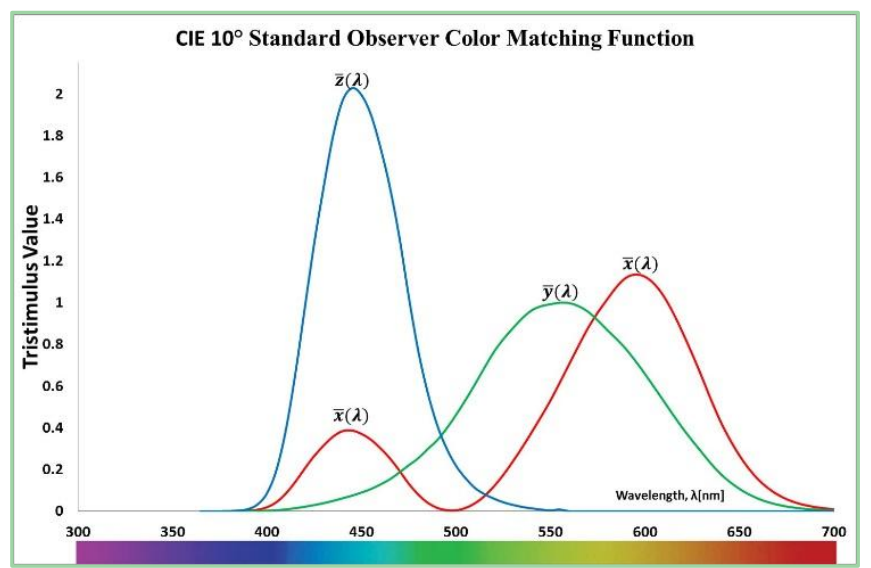

Fig. $4.10^{\circ}$ Spectral response curves corresponding to the CIE definition of the 1964 standard observer.

Fig. 4. refers to the $10^{\circ}$ standard observers CIE's color matching functions denote by $\bar{x}_{10}(\lambda), \bar{y}_{10}(\lambda)$ and $\bar{z}_{10}(\lambda)$ which is referred to the red, green and blue color matching functions, respectively. The CIE tristimulus values of $X_{10}, Y_{10}$ and $Z_{10}$ are the numerical descriptions of the chromatic response of the observer. The value also refers to the spectral sensitivity curves of three linear light detectors and are known as the CIE standard observer [11].

\subsection{The Illuminant of a Light Source}

An illuminant is a radiation with a defined relative spectral power distribution. It also refers to a graphical representation of the light source under which the samples are viewed. The two most commonly used standard illuminants are CIE standard illuminant A and CIE standard illuminant D65.

The CIE is defined D65 as a normal daylight and with correlated color temperature of approximately 6500K. Standard illuminant D65 is used in all colorimetric calculations which representing a daylight unless there are specific different illuminants are mentioned. As for CIE standard illuminant A, at a temperature of approximately $2856 \mathrm{~K}$, it is referred to more a domestic and tungsten-filament lighting with Planckian 
radiator. For colorimetry involving the use of the incandescent lighting, this specification illuminant should be utilized unless it was mentioned to use others for the specific purposes [12]. The analysis in detecting cyanosis could be affected by lighting color and environment [13]. For a further simplification, the scope of the illuminant for $\mathrm{I}_{\text {In }}$ in Fig. 2 is set to approximately constant in all range of the visible wavelength. This simplification represents there is the constant illuminant use in this study and any factors that affected the illuminant has been neglected.

\subsection{The Transmission Spectra of $\mathrm{HbO}_{2}$ and $\mathrm{Hb}$}

The absorption properties in the dermis are dominantly determined by $\mathrm{HbO}_{2}$ and $\mathrm{Hb}$. The absorption spectra of $\mathrm{HbO}_{2}$ and $\mathrm{Hb}$ are depicted in Fig. 5. From the absorption spectra of $\mathrm{HbO}_{2}$ and $\mathrm{Hb}$ obtained in Fig. 6 , the transmission spectra of both spectra are calculated based on Beer's Law. The transmission of $\mathrm{HbO}_{2}$ and $\mathrm{Hb}$ can be written in the inverse relationship of the absorbance [14].

$$
\begin{gathered}
\operatorname{Absorbance}(A)=\log _{10}\left(\frac{1}{T}\right)=\log _{10}\left(\frac{I_{0}}{I}\right) \\
\% T=100\left(\frac{I}{I_{0}}\right)=e^{-\varepsilon l c},
\end{gathered}
$$

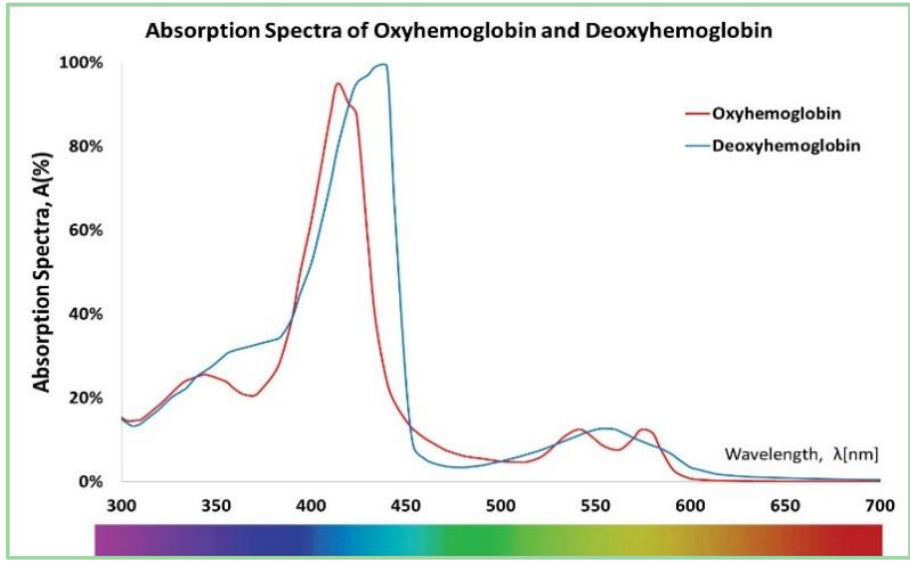

Fig. 5. Absorption spectra of $\mathrm{HbO}_{2}$ and $\mathrm{Hb}$. Original database are from Scott Prahl [15]

where $I_{0}$ is the incoming light, $I$ is the output light, $e$ is 2.718, $\varepsilon$ is a molar absorptivity, $l$ is a path length and $c$ is concentration. From (5), an absorbance is rated from 0 to 2 . At 0 absorbance unit at some wavelength means that no light of that particular wavelength has been absorbed or $100 \%$ of transmittance. In the other hand, 1 absorbance means only $10 \%$ of light has been transmitted. $\% \mathrm{~T}$ refers to the transmission of light in percentage. To tackle this issue, the transmission spectra of Ray 2a is obtained as the spectral transmission graph shown in Fig. 6.

In the red wavelength region, the reflectance or the amount of reflected light is higher than in the other wavelength regions. Furthermore, it shows that the Ray 2a which contains the transmission of oxyhemoglobin reflects more light in the range of orange and red wavelength regions while absorbs light in the green, blue, indigo, and violet wavelength regions. The transmission graph in this figure shows the oxygenated blood is more red than the deoxygenated blood.

On the other hand, as can be seen, the transmission spectra of Ray $2 \mathrm{~b}$ are the transmission spectra of deoxyhemoglobin. It shows the high amount of reflected light in the blue wavelength region as depicts in Fig. 7 whereas the reflectance light in red wavelength is low and almost all the green wavelength region being absorbed. The spectra of Ray $2 \mathrm{~b}$, which transmits the deoxygenated blood looks bluer that the oxygenated 
ray in Ray 2 a.

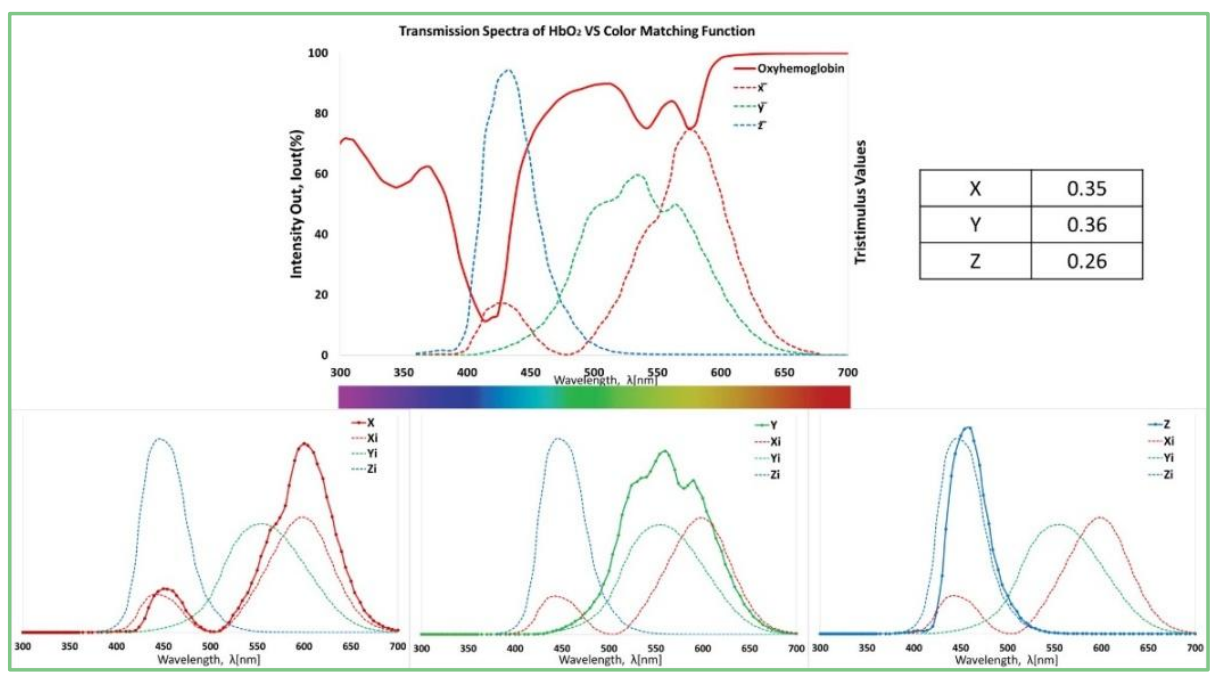

Fig. 6. Transmission spectra of oxyhemoglobin versus color matching function.

The mathematics can be applied to describe the blue coloration of cyanosis and non-cyanosis in tristimulus values of $X, Y$ and $Z$ where $P(\lambda)$ is the spectral distribution of light stimulus and $k$ is a normalizing constant by calculate the integrals by a stepwise integration.

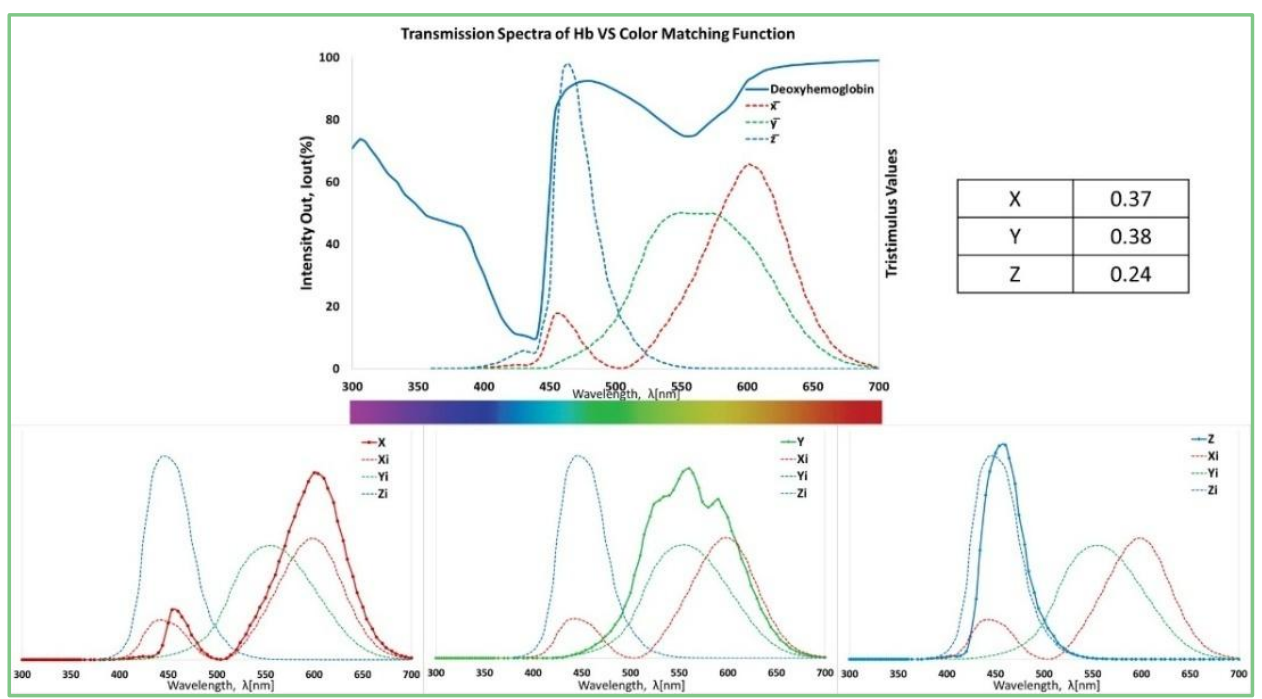

Fig. 7. Transmission spectra of deoxyhemoglobin versus color matching function.

$$
\begin{aligned}
& X=k \int P(\lambda) \cdot \bar{x}_{10}(\lambda) d \lambda \\
& Y=k \int P(\lambda) \cdot \bar{y}_{10}(\lambda) d \lambda \\
& Z=k \int P(\lambda) \cdot \bar{z}_{10}(\lambda) d \lambda
\end{aligned}
$$

For $\mathrm{HbO}_{2}$ and $\mathrm{Hb}, P(\lambda)$ is given by

$$
\begin{gathered}
I_{I n}(\lambda)=I_{I n}(\lambda) \cdot T_{H_{b O}}(\lambda) \\
I_{I n}(\lambda)=I_{I n}(\lambda) \cdot T_{H b}(\lambda),
\end{gathered}
$$


where $T_{\mathrm{HbO}_{2}}(\lambda)$ and $T_{\mathrm{Hb}}(\lambda)$ is the transmission spectral of $\mathrm{HbO}_{2}$ and $\mathrm{Hb}$ respectively as in Fig. 6 and Fig. 7, $I_{I n}(\lambda)$ is the relative irradiance of illumination and $k$ is refers to the $\mathrm{CIE} 10^{\circ}$ standard observer's constant [16].

\subsection{Quantifying a Cyanosis}

As for the next step, the calculation for determining the cyanotic and non-cyanotic skin has been made according to CIE $\mathrm{L}^{*} \mathrm{a}^{*} \mathrm{~b}^{*}$ system using the following formula as for the accurate determination of cyanotic and non- cyanotic colors and color differences [17]:

$$
a^{*}=500 *\left(\left(X^{\frac{1}{3}}\right)-\left(Y^{\frac{1}{3}}\right)\right), b^{*}=200 *\left(\left(Y^{\frac{1}{3}}\right)-\left(Z^{\frac{1}{3}}\right)\right), L^{*}=\left(116 *\left(Y^{\frac{1}{3}}\right)\right)-16
$$

The calculation of the coordinate $\left(\mathrm{L}^{*}, \mathrm{a}^{*}, \mathrm{~b}^{*}\right)$ are from the $(\mathrm{X}, \mathrm{Y}, \mathrm{Z})$ of the given light stimulus and the white point of $(\mathrm{Xi}, \mathrm{Yi}, \mathrm{Zi})$. This is the reason CIE $\mathrm{L}^{*} \mathrm{a}^{*} \mathrm{~b}^{*}$ color space has a function for correcting the chromatic adaptation to the white point and is usually present as the object color and displays [18]. The CIE $\mathrm{L}^{*} \mathrm{a}^{*} \mathrm{~b}^{*}$ color space system is the most widely used space for measuring color. In this color wheel, $\mathrm{L}^{*}$ indicates lightness and $\mathrm{a}^{*}$ and $\mathrm{b}^{*}$ indicate the chromaticity coordinates, with $\mathrm{a}^{*}$ range from the red $(+100)$ to green $(-100)$ axis while $b^{*}$ range from the yellow $(+100)$ to blue $(-100)$. The color values of $\mathrm{HbO}_{2}$ and $\mathrm{Hb}$ can be represented in the $\mathrm{L}^{*} \mathrm{a}^{*} \mathrm{~b}^{*}$ three-dimensional color space as shown in Fig. 8 displaying in $\mathrm{a}^{*} \mathrm{~b}^{*}$ plane. In the figure, both $\mathrm{HbO}_{2}$ and $\mathrm{Hb}$ is closer to the $+\mathrm{a}^{*}$ axis, and $\mathrm{HbO}_{2}$ is more to the red than $\mathrm{Hb}$. As for the chroma or the saturation, the $\mathrm{Hb}$ is "paler" than the $\mathrm{HbO}_{2}$ 's color. The word "paler" here indicates the degree of differences in the chroma between $\mathrm{HbO}_{2}$ and $\mathrm{Hb}$. The chromaticity values of $\mathrm{L}^{*} \mathrm{a} * \mathrm{~b}$ for $\mathrm{HbO}_{2}$ and $\mathrm{Hb}$ indicates in Fig. 8. prove that the proposed method give the significant different in color values of $\mathrm{HbO}_{2}$ and $\mathrm{Hb}$. The $\mathrm{a}^{*} \mathrm{~b}^{*}$ values, representing the hues change significantly between these two main chromophores which provide the bluish cyanosis effect to the skin.

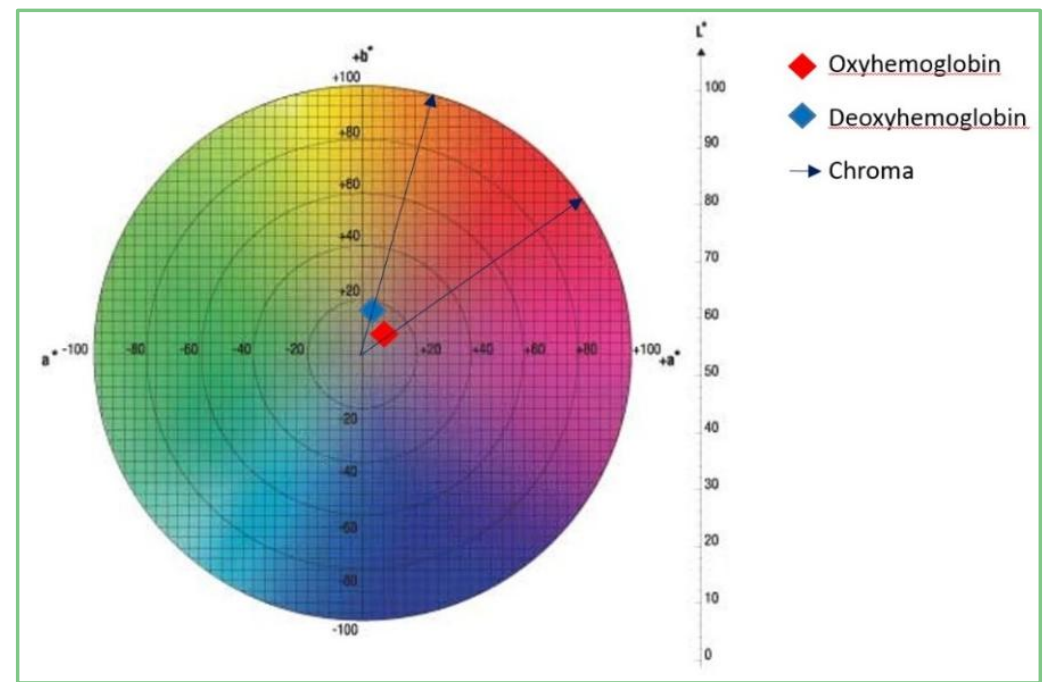

Fig. 8. a* and $\mathrm{b}^{*}$ chromaticity for $\mathrm{HbO}_{2}$ and $\mathrm{Hb}$ plot with the color diagram in the background.

\section{Conclusion}

In this paper, a new conceptual development of cyanosis determination and assessment method is presented relying on the on the measurement of the difference in the lowest value of the transmission spectra of $\mathrm{HbO}_{2}$ and $\mathrm{Hb}$. The lowest wavelengths of $\mathrm{HbO}_{2}$ and $\mathrm{Hb}$ obtained at $424 \mathrm{~nm}$ and $440 \mathrm{~nm}$ 
respectively. However, the recognition of cyanosis skin based on wavelength's difference is not practical in a modern clinical practice. Thus, another quantitative measurement using color matching function is introduced and the objective of cyanotic and non-cyanotic skin color measurement based on CIE color space values $\left(\mathrm{L}^{*}, \mathrm{a}^{*}\right.$ and $\mathrm{b}^{*}$ ) are determined. This paper highlights two major types of clarification: the oxygenated blood is redder than the deoxygenated blood while the deoxygenated blood is bluer than oxygenated blood.

In future work, an actuator for a cyanosis baby manikin using the modeling presented in this article will be developed.

\section{Acknowledgment}

The author of Nur Fatihah Azmi is funded by the funds from Ministry Education of Malaysia and Universiti Teknikal Malaysia Melaka. The author would like to acknowledge the Department of Industrial Design, Eindhoven University of Technology for their excellent support throughout this research activity. Also the acknowledgment for Prof. Dr. Sidarto Bambang Oetomo from Máxima Medical Centre for his support and contribution towards this project.

\section{References}

[1] Frommelt, P. C., \& Frommelt, M. A. (1996). Cyanosis. Practical Strategies In Pediatric Diagnosis and Therapy, 166-180.

[2] Morgan, E. A., \& Brown, A. (1935). Cyanosis of the new-born. JAMA J. Am. Med. Assoc., 105(3), 1085-1088.

[3] Lanzkowsy, P. (2015). Neonatal cyanosis and arterial oxygen saturation. J. Pediatr., 2, (319-324).

[4] Cyanosis. Central Cyanosis. Retrieved from the website: http://www.nhs.uk/Conditions/cyanosis)

[5] Blake, D. (2010). Do we assess 'colour' appropriately using the Apgar score? J. Neonatal Nurs., 16(4), 184-187.

[6] Steinhorn, R. H. (2008). Evaluation and management of the cyanotic neonate. National Institute of Health, 9(3), 169-175.

[7] Lawrence, M. H. K. (1990). How much reduced hemoglobin is necessary to generate central cyanosis. Chem. Mater., 97(1), 182-185.

[8] Peters, P., Delbressine, F., \& Feijs, L. (2014). Designing preterm neonatal cyanosis simulation. IWBBIO. 1325-1337.

[9] Zohdi, T. I., \& Kuypers, F. A. (2006). Modelling and rapid simulation of multiple red blood cell light scattering. J. R. Soc. Interface, 3(11), 823-831.

[10] Smith, G. S. (2005). Human color vision and the unsaturated blue color of the daytime sky. American Journal of Physics, 73(7), 590.

[11] Stockman, A., \& Sharpe, L. T. (1999). Cone spectral sensitivities and color matching. Color Vis. from Genes to Percept, 1855, 53-87.

[12] Hsia, J. J., Mielenz, K. D., \& Moore, J. R. (1998). CIE Standard Illuminants for Colorimetry. ISO 10526:1999/CIE S005/E-1998. Retrieved from the website: http://www.cie.co.at/publ/abst/s005.html

[13] S. J. Dain, "The visual recognition of cyanosis and the influence of lighting and color vision," in Advances in Dermatology Research, pp. (132-139).

[14] Trg̃er, F. (2005). Optical materials and their properties. Springer Handbook of Lasers and Optics, 1st ed. Springer Science+Business Media. 249-372.

[15] Scott, P. S. J. (2015). Optical absorption and emission data. Retrieved from the website: http://omlc.ogi.edu 
[16] Ohno, Y. (2000). CIE fundamentals for color measurements. History, 1-6.

[17] Pascale, D., et al. (2002). Color Vision and Colorimetry: Theory and Applications, 4th Edition.

[18] Ohno, Y. (2000). CIE fundamentals for color measurements. Proceedings of IS\&T NIP16 Conference (pp. 1-6). Vancouver, Canada.

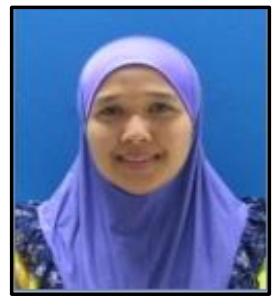

Malaysia (BEM).
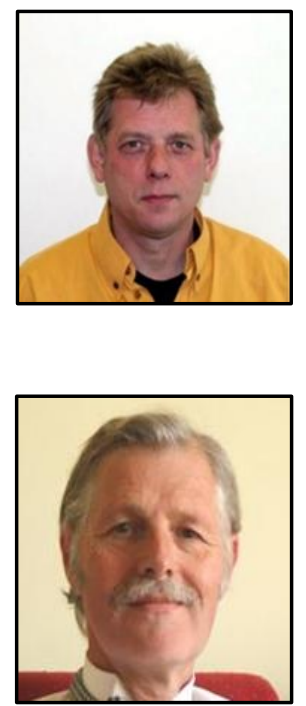

Frank Delbressine received his Ph.D. from TU/e in mechanical engineering in 1989. His main research interests are in medical simulation, wearables and autonomous vehicles. Currently, he is an assistant professor at ID Department, TU/e, NL.

Loe Feijs received his B.E and M.E in electrical engineering from TU/e. In 1990, he obtained his Ph.D. in computer science of TU/e. Before that, he worked in Philips as a scientist and software developer for about 14 years. In 1994, he was appointed a part-time professor of TU/e Mathematics and Computer Science. At present, he is a full professor at ID Department, TU/e, NL. His current research interests include creative programming, medical simulation, wearables and mathematical modeling. 